\title{
Standardization of pitch-range settings in voice acoustic analysis
}

\author{
Adam P. Vogel and Paul Maruff \\ University of Melbourne, Melbourne, Victoria, Australia \\ Peter J. SNyder \\ Rhode Island Hospital, Providence, Rhode Island \\ and Warren Alpert Medical School, Brown University, Providence, Rhode Island \\ AND \\ JAMES C. MundT \\ Healthcare Technology Systems, Madison, Wisconsin
}

\begin{abstract}
Voice acoustic analysis is typically a labor-intensive, time-consuming process that requires the application of idiosyncratic parameters tailored to individual aspects of the speech signal. Such processes limit the efficiency and utility of voice analysis in clinical practice as well as in applied research and development. In the present study, we analyzed 1,120 voice files, using standard techniques (case-by-case hand analysis), taking roughly 10 work weeks of personnel time to complete. The results were compared with the analytic output of several automated analysis scripts that made use of preset pitch-range parameters. After pitch windows were selected to appropriately account for sex differences, the automated analysis scripts reduced processing time of the 1,120 speech samples to less than $2.5 \mathrm{~h}$ and produced results comparable to those obtained with hand analysis. However, caution should be exercised when applying the suggested preset values to pathological voice populations.
\end{abstract}

Acoustic analysis of the voice requires the selection of parameter settings specific to sample characteristics, such as intensity, duration, frequency, and filtering. Historically, acoustic experiments have relied on small sample sizes, for which the laborious use of idiosyncratic parameters for individual samples on a case-by-case basis was feasible (Kent \& Read, 2002). As a result, most voice acoustic studies lack standardized or automated analytical procedures (Titze, 1994). Although perceptual evaluation of the voice during clinical investigations remains the primary method for assessing vocal change (Kent \& Read, 2002), Carding, Carlson, Epstein, Mathieson, and Shewell (2001) have identified numerous limitations of such analysis, citing poor reliability of analyses within and among raters (Bassich \& Ludlow, 1986), disparity in the design of the available perceptual rating scales, variability of the human voice, and difficulties in interpreting complex acoustic signals as contributory factors. Despite these limitations, it must be acknowledged that some clinical populations may not be suitable for standardized analysis procedures. Voice disorders, such as glottal fry (where the voiced speech signal may contain aperiodic signals) or atypical frequency profiles (e.g., puberphonia), may also be inappropriate for generic analysis procedures and thus would require a combination of perceptual and idiosyncratic evaluation.
The need for objective and repeatable evaluation of vocal change in large, generalizable experimental settings calls for greater accountability and quantitative judgment in voice acoustic research (Eskenazi, Childers, \& Hicks, 1990; John, Sell, Sweeney, Harding-Bell, \& Williams, 2006; Kent, 1996). Relative to procedures based on perceptual judgments, standardized acoustic analysis has the advantage of providing objective and repeatable measures of vocal change.

Current procedures for acoustic voice analysis typically require idiosyncratic specifications of pitch-range settings that determine window length, selection of intensity cutoffs, hand splicing of sound files, and expert knowledge of particular software and hardware configurations. Such case-by-case analytic procedures are time and resource intensive and are an impediment to the growing need for fast and accurate voice analysis. Development of standardized, automated procedures could streamline this process, thus reducing the time, cost, and need for handson intervention.

Standardizing the selection of the pitch-range settings for measuring particular acoustic properties using known characteristics of the speakers could provide a means for enhancing the efficiency of the analytic process. Window frame lengths determine how pitch contours are displayed

A. P.Vogel, avogel@cogstate.com 
and computed, and the application of inappropriate frame properties can result in inaccurate and unreliable data. However, to date, very few studies have evaluated the effects of systematically altering window frame length or pitch-range settings on large batches of sound files and compared them with idiosyncratically selected gold standards. Attempts to employ automated pitch extraction and window length algorithms are being refined (Atkinson, Kondoz, \& Evans, 1995; Fette, Gibson, \& Greenwood, 1980; Karnell, Scherer, \& Fischer, 1991; Rabiner, 1977), but this remains a complicated process. Until fast and accurate techniques are developed to facilitate large-batch processing of voice files, preset frame sizes designed for distinct population groups will continue to be required. Fortunately, tailored techniques that adapt to subtle pitch variations within an utterance are generally unnecessary, because the variability within an utterance is usually within one octave above or below the average pitch in a typical sample (Rabiner, 1977).

A number of studies have addressed analytic processes related to window size or type (Fette et al., 1980; Takagi, Seiyama, \& Miyasaka, 2000), signal length requirements (Scherer, Vail, \& Guo, 1995), timing (Green, Beukelman, \& Ball, 2004), prosodic measures (Hirst, 2002), and appropriateness of stimuli used to elicit acoustic measures (Zraick, Wendel, \& Smith-Olinde, 2005). Calls for better standardization and automation of voice analysis are prevalent in the literature, yet significant technical limitations remain. Consequently, objective studies of the voice continue to be dominated by relatively small, clinical experiments that do not require rapid processing of large batches of acoustic data. Understandably, there is little incentive to develop or apply generic analysis settings under such circumstances, and the use of such settings may not be appropriate for all acoustic measures. For example, measures of perturbation, such as jitter and shimmer, may vary within and among analysis tools, because voices that violate assumptions of periodicity often require case-by-case evaluation (Karnell, Hall, \& Landahl, 1995; Perry, Ingrisano, \& Scott, 1996; Titze \& Liang, 1993). Similarly, extraction of error-free pitch measures from a voice that varies within a short period of time and differences between speakers due to sex or emotional state can be problematic (Mendoza, Muñoz, \& Valencia Naranjo, 1996; Mueller, 1997). Consequently, it is important that window frame lengths be kept short, because fast excursions of pitch should not be smoothed over (Gerhard, 2003); however, they should be long enough to complete enough cycles of the periodic waveform to provide data for analysis (Fette et al., 1980; Karnell et al., 1991).

It is advantageous to the researcher that some acoustic measures appear to retain levels of consistency, despite alterations in analysis methodology, especially those relating to the fundamental frequency $(f 0)$. $f 0$ has demonstrated robustness as an acoustic parameter in trials comparing software configurations (Kent, Vorperian, \& Duffy, 1999; Takagi et al., 2000; Vogel \& Maruff, 2008), environments with poor signal-to-noise ratios (Deliyski, Shaw, \& Evans, 2005a), acquisition environments (Deliyski, Evans, \& Shaw, 2005), and sampling rates (Deliyski,
Shaw, \& Evans, 2005b). Aspects of motoric timing during speech production, such as measures of pause frequency and speaking rate, have also been found to be important, replicable measures (Cannizzaro, Harel, Reilly, Chappell, \& Snyder, 2004). On the basis of existing literature and past experiences, a limited range of upper and lower bounds for defining pitch ranges for voice acoustic analysis appears to be adequate for "normal" vocal populations. Development of automated or semiautomated processes that would allow widespread application of generic pitch ranges, in the context of voice acoustic analysis, has the potential to lessen the use of idiosyncratic analytical settings. Evaluation of such a process, however, requires comparison with results obtained through laborious, resource-intensive, gold-standard practices of individually analyzed files. The use of automated processes for the analysis of large batches of speech data could dramatically decrease the time and resources required for acoustic studies and could improve the reliability and repeatability of study results. Considering a single, fixed window frame length has been shown to be unsuitable for all speech samples (Rabiner, 1977). Therefore, the present study investigated the potential use of generic pitch-range and window frame settings tailored to the sex of the speaker. The resulting acoustic measures are compared with those obtained through optimized gold-standard methods resulting from individual, case-by-case analysis.

\section{METHOD}

\section{Participants}

Twenty participants ( 10 female, 10 male; mean age $=40.5$ years) who had participated in a methodology study investigating speech changes associated with treatment for depression were selected randomly from a cohort of 35 . Demographics, clinical characteristics, participation criteria, and study procedures are described in Mundt, Snyder, Cannizzaro, Chappie, and Geralts (2007).

\section{Data Collection}

Speech samples produced in response to automated elicitation procedures were obtained over a standard office telephone using an interactive voice response system (Mundt et al., 2007). Performance of the speech elicitation tasks produced samples of free speech (each participant extemporaneously discussed his/her recent physical, emotional, and general functioning for a period lasting around $1 \mathrm{~min}$ ), prolonged vowels (/a:/, /ae:/, /u:/,/i:/), and reading of a standard passage (grandfather passage, 175 syllables). Validation of the telephony-based procedures for eliciting voice acoustic measures comparable to those obtained in a laboratory setting have been published (Cannizzaro, Reilly, Mundt, \& Snyder, 2005).

Each study participant produced 56 speech samples, generating a total of 1,120 recordings for vocal acoustic analysis. Each speech sample was scored individually by hand, requiring approximately 350-420 total hours of personnel time. The case-by-case, handscored acoustic measures provided the gold standards for evaluating the accuracy of the automated scoring procedures described below.

\section{Measures}

The primary measures of interest for the present investigation were $f 0, f 0$ standard deviation $(S D)$, and $f 0$ coefficient of variation (CV). These measures were selected for their robust analytical potential, because frequency measurements have been shown to be resistant to acoustic artifaction and are largely independent of signal quality. Frequency profiles were calculated from the samples previously mentioned. $f 0$, and its corresponding $S D$ and $\mathrm{CV}$, were derived from all samples. 


\section{Procedure and Acoustic Analysis}

All speech samples were segmented and analyzed using Praat (version 5.0.32; Boersma \& Weenink, 2008), which employs a usersupplied estimate of analysis window length. Silences were removed from the start and end of the sustained vowel samples, and each sample was truncated by $1.5 \mathrm{sec}$ on each side of its temporal midpoint. The other speech samples were not truncated. In order to determine window length, we considered two primary parameters. Time step is a measurement interval (frame duration) in seconds and is calculated (when time step is set to 0 ) by dividing 0.75 by the value of the pitch floor (e.g., if the pitch floor is $75 \mathrm{~Hz}$, the time step equals $0.1 \mathrm{sec}$, prompting Praat to compute 100 pitch values per sec). Pitch floor determines the length of the analysis window and represents the lowest $f 0$ targeted within each sample. For calculation of pitch, the window should be long enough to contain three periods (for pitch detection); for example, for a pitch floor of $75 \mathrm{~Hz}$, the window is effectively $3 / 75=0.04 \mathrm{sec}$ long. Increasing the time step speeds up the editor window; however, it may lead to undersampling of the pitch and formant curves, which, in turn, influences the accuracy of selected measures. The pitch ceiling is a postprocessing step that ignores candidates above the prescribed setting and promotes the most efficient use of available data. A summary of the complete multiparameter algorithm for calculating the $f 0$, as it is implemented into the speech analysis and synthesis program Praat, is described in Boersma (1993).

In Praat, pitch-range settings are the most important parameters in pitch analysis. As described, the pitch floor determines the window length and the pitch ceiling restricts the values that are recruited during the analysis. Therefore, the question of automatically defining optimal values for floor and ceiling is not trivial, and the output can differ, depending on the values used. The pitch-range/window-range settings could be displayed in units of time; however, for ease of translation, the present study has maintained units of measurement in hertz. On the basis of professional experience, recommended software settings, and clinical impressions of pitch variation between male and female speakers, we considered pitch floor values $(50,70$, $100 \mathrm{~Hz})$ in conjunction with five pitch ceiling values $(250,300,500$, $600,625 \mathrm{~Hz}$ ) as potential sex-specific, generic frequency window settings for automating acoustic analysis. Pitch floor settings dictate that candidates below this frequency are not recruited; similarly, the pitch ceiling ensures that candidates above this frequency are ignored. All $f 0$ plots were produced by an autocorrelation algorithm. We evaluated the nine resulting sets of acoustic parameters defining 13 potential floor/ceiling pitch-range settings: 50-250, 50-300, 50$500,50-600,70-250,70-300,70-500,70-600,70-625,100-250$, $100-300,100-500$, and $100-600 \mathrm{~Hz}$. The results from each setting were compared with data derived from the gold-standard settings, which were based on unique sample characteristics identified during hand scoring of the data reported in Mundt et al. (2007).

The complete set of 1,120 speech samples (20 participants, each providing 56 samples) was analyzed using each of the 13 pitch-range settings identified above. Thus, a total of 14,560 analytic cycles were performed through the use of automated batch processing of the speech samples. The derived $f 0, f 0 S D$, and $f 0 \mathrm{CV}$ measures obtained from each cycle were stored and compared with the goldstandard measures obtained from the individually hand-scored samples. Gold-standard measures were determined by visually inspecting the waveform of each sound file and selecting periodic parts of the signal. It is easy for speech scientists to visually identify parts of the signal that conform to a regular pattern; however, this is a difficult task for software. The signal characteristics (e.g., frequency range of sample) were identified manually and used for analysis. This process is very time consuming, but currently is considered the most accurate method of acoustic analysis.

\footnotetext{
Statistical Analysis

One-way ANOVA was used to analyze statistical differences of data sets, and differences among groups were assessed by Dunnett's method, which compares independent variables (generic pitch settings) with control (gold-standard) data using commercially
}

Table 1

Acoustic Measures Obtained Using Gold-Standard Case-by-Case Pitch Settings

\begin{tabular}{|c|c|c|c|c|c|}
\hline & \multicolumn{2}{|c|}{ Males } & \multicolumn{2}{|c|}{ Females } & \multirow[b]{2}{*}{$p$ value } \\
\hline & $M$ & $S D$ & $M$ & $S D$ & \\
\hline$f 0 M$ & 114.74 & 25.79 & 170.19 & 20.51 & $<.0001$ \\
\hline$f 0 S D$ & 7.26 & 6.46 & 11.37 & 10.54 & $<.0001$ \\
\hline$f 0 \mathrm{CV}$ & 0.06 & 0.05 & 0.07 & 0.07 & .2854 \\
\hline
\end{tabular}

Note-Males, $N=10$; Females, $N=10$.

available statistics software (JMP 7.0 Statistical Discovery, SAS Institute, Cary, NC). Effect sizes were calculated using Cohen's $d$; $p$ values $<.05$ were considered statistically significant.

\section{RESULTS}

Data obtained via gold-standard analysis were grouped by sex (Table 1). Independent-group $t$ tests were employed to compare the means of each measure. Results revealed significant differences between males and females $(p<$ $.05)$ for $f 0$ and $f 0 S D$ values.

When analyzed via gold-standard practices, those measures that varied significantly between sexes were then examined using the generic pitch-range settings. Statistical differences between gold-standard techniques and trial pitch-range setting groups were analyzed by one-way ANOVA and Dunnett's multiple comparisons test using JMP Statistical Discovery. Resulting differences between gold-standard methods and specific trial pitch frames were analyzed further by Student's $t$ test.

One-way ANOVA of the $f 0$ obtained by each of the pitch-range settings revealed significant variance among groups for both female $[F(4635)=10.94, p<.0001]$ and male $[F(3910)=92.1, p<.0001]$ groups. Similar findings were observed on measures of $f 0 S D$ [female, $F(4635)=67.6, p<.0001$; male, $F(3910)=166.74, p<$ .0001 ] and $f 0 \mathrm{CV}$ [female, $F(4635)=66.77, p<.0001$; male, $F(3910)=150.92, p<.0001]$.These results suggest that $f 0, f 0 S D$, and $f 0 \mathrm{CV}$ values require specific pitch-range settings.

Dunnett's multiple comparison method revealed significant differences between methods on the mean scores of $f 0, f 0 S D$, and $f 0 \mathrm{CV}$ for both female (Table 2) and male (Table 3) groups.

Data in Tables 2 and 3 illustrate the degree of overall variability inherent in the selection of frame range settings, despite controlling for a number of variables. We repeatedly analyzed all 1,120 speech samples by using each pitch-range setting with the same software program and analysis scripts. Because only one aspect of the study was modified during each analysis cycle, all observed variance in $f 0$ was due to manipulation of pitch-range settings.

Using $f 0$ as a guide, we identified a number of generic settings that appeared to provide accurate estimates of pitch; however, when the data were broken down by task, very few settings yielded equivalent results across all stimuli and measures in comparison with data derived from handselected settings. Speech samples that fell outside of the prescribed generic pitch-range settings were not analyzed 

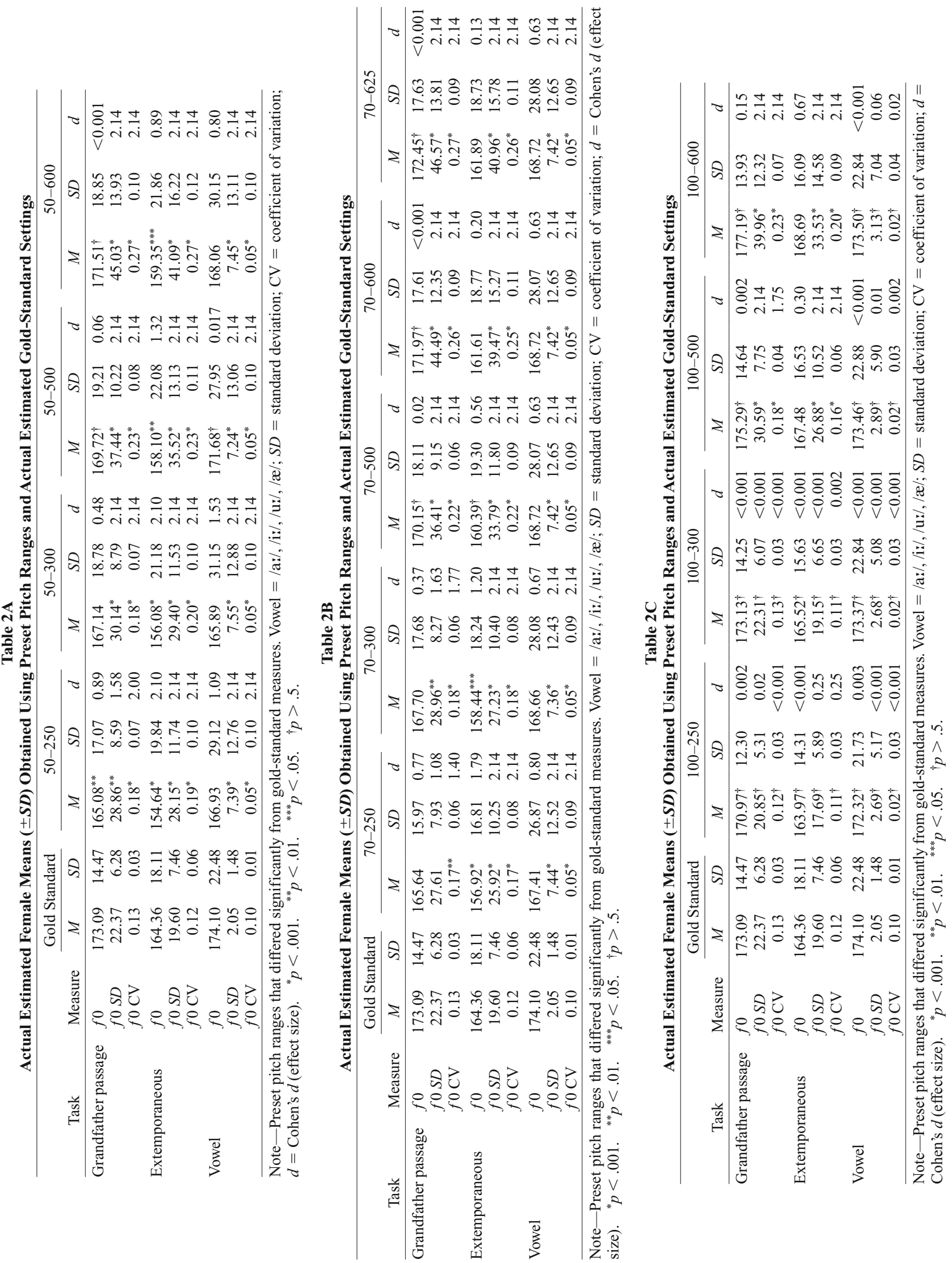

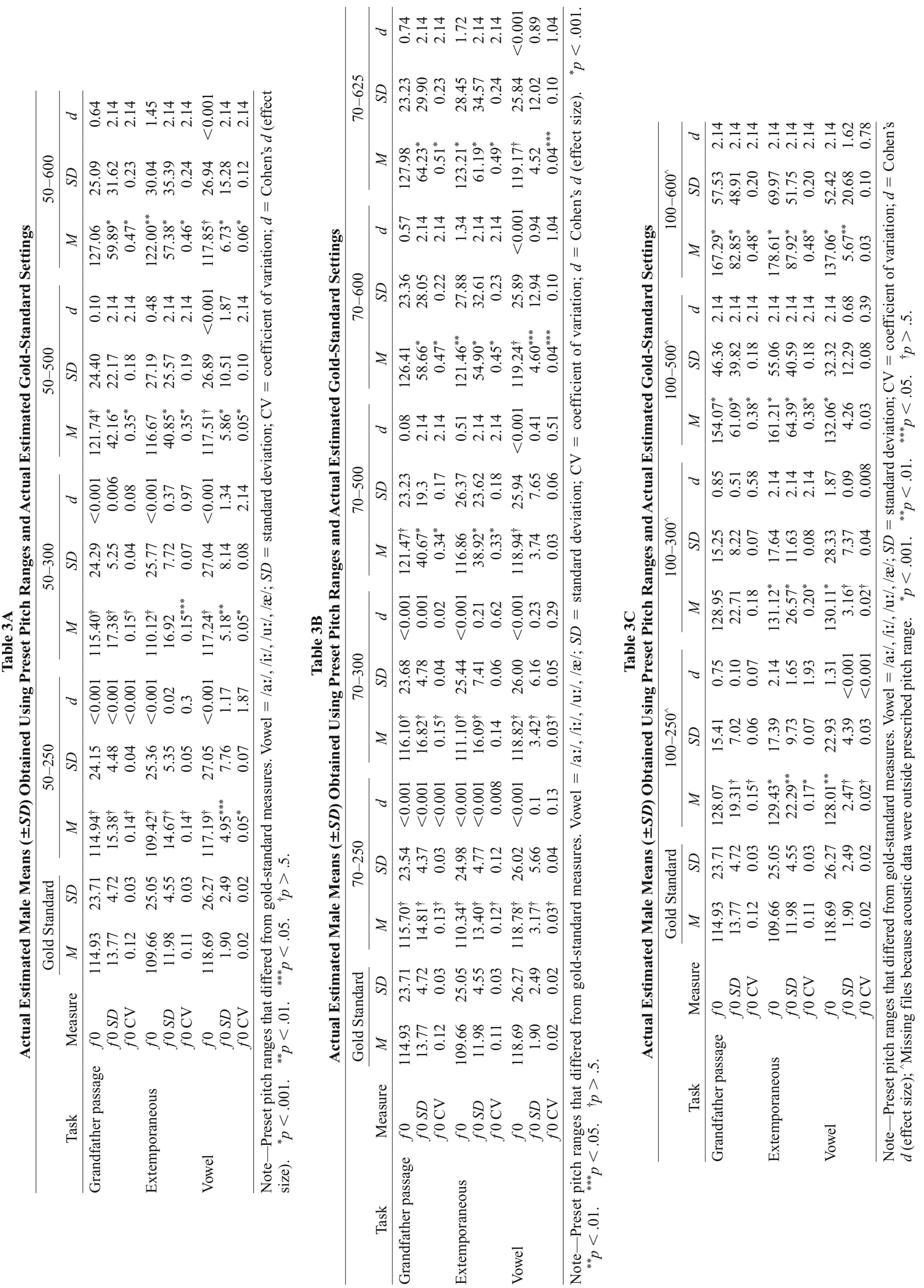
automatically, and consequently were not included in the results. All female voice data were analyzed without exclusion; however, 17 files were excluded within the male voice batch when the pitch floor was $100 \mathrm{~Hz}$. In light of these issues, of specific interest are the experimental conditions that, when broken down by sex and task, appear to be suitable for large-batch analysis of all acoustic data. Statistical differences and significant effect sizes were not observed between gold-standard techniques and specific pitch-range lengths for pitch ranges of $100-300 \mathrm{~Hz}$ and $100-250 \mathrm{~Hz}$ for females and $70-250 \mathrm{~Hz}$ for males.

\section{DISCUSSION}

The use of sex-specific, generic pitch-range lengths on more than 1,100 speech files in an automated analysis script produced results statistically equivalent to those obtained from idiosyncratic, individualized, gold-standard practices. A strong relationship was observed among window ranges determined by pitch-range settings that adapted to the higher and lower frequencies introduced by the sex of the speaker. Specifically, we identified a clear set of pitch-range settings suitable for male participants that encompassed low pitch floors (e.g., $70 \mathrm{~Hz}$ ) and midlevel pitch ceiling (e.g., $250 \mathrm{~Hz}$ ). Similarly, a pitch floor setting of $100 \mathrm{~Hz}$ and ceiling settings of 250 and $300 \mathrm{~Hz}$ for female speakers yielded results comparable to those obtained with current gold-standard practices. Identification of speaker sex, prior to analyzing the voice samples to obtain acoustic measures such as $f 0$, permits part-individualization of analysis procedures and promises to increase the efficiency and speed of analysis in comparison with current hand-scoring procedures.

These findings underscore the value of considering generic window lengths as a means of expediting large-batch voice acoustic analysis for both male and female populations. Traditional analysis methods typically rely on laborintensive, individually selected settings or slow adaptive analysis algorithms based on variation in frequency within the speech signal (Karnell et al., 1991; Morris \& Brown, 1996; Rabiner, 1977). In the present study, hand scoring of the 1,120 voice files required roughly 10 work weeks of personnel time. Adapting the analysis settings on the basis of known characteristics of the speaker offers an alternative to laborious, idiosyncratic analyses. Such adaptive techniques must be validated in acoustic environments that require fast and accurate analysis of several thousand speech files. Automated analysis of the same 1,120 voice files using sex-specific pitch-range settings required an average of only 5 sec per file.

The relationship between frame lengths and the $f 0$ of a speaker is complex, due to the inherent variations of frequency profiles (Hirose, Fujisaki, \& Seto, 1992), but, because it requires extensive expertise and time, managing speaker-specific analysis settings individually is impractical for large volumes of data. If the pitch floor is set too low, very fast $f 0$ changes are missed; if it is set too high, low $f 0$ values are neglected. However, by separating populations into distinct vocal groups (e.g., male and female), a set of cohort-specific settings can be applied.
Such procedures could greatly reduce the labor-intensive processes used in current practices.

Inherent variability of the human voice precludes the use of generic frame lengths in all populations, but standardized sex-specific pitch ranges may be suitable for a wide range of "typical" frequency profiles. Individuals with frequency profiles outside expected levels may not be analyzed accurately by using automated acoustic analysis. However, effectively screening participants and establishing reasonable restrictions on the recording environment and software selection could permit effective application of automated and standardized acoustic analyses.

Clinically and within the literature, consideration of the prescriptive aspects related to both procedure and analysis of voice acoustics is undertaken rarely, despite calls for standardization and consensus. Titze (1994) put forth arguments for the implementation of standardized recording techniques and analysis settings, stating that simplification of techniques would allow for a reduction in the number of processes involved. Titze also suggested that standardization can lead to conservation of resources - in this case, time, money, and expertise.

In the present study, we used generic pitch-range settings for 14,560 analysis cycles to obtain widely used acoustic measures, such as $f 0$, in individuals with periodic speech signals. The measures obtained demonstrated that the use of appropriately selected, sex-specific pitch settings yield results comparable to measures obtained from individualized analysis procedures. The acoustic data were analyzed in a fraction of the time that individual analyses would have required, promising new opportunities for researchers to improve the accuracy and reliability of large studies investigating vocal acoustics, while decreasing the labor and time required for current practices.

\section{AUTHOR NOTE}

Data collection for the present study was supported by Small Business Innovation Research Grant R43MH68950 from the National Institute of Mental Health. We acknowledge Amy Fredrickson for her invaluable statistical advice and Paul Kukiel for his work on the analysis scripts. Address correspondence to A. P. Vogel, University of Melbourne, Level 7/21, Victoria Street, Melbourne, VIC 3000, Australia (e-mail: avogel@ cogstate.com).

\section{REFERENCES}

Atkinson, I. A., Kondoz, A. M., \& Evans, B. G. (1995). Pitch detection of speech signals using segmented autocorrelation. Electronics Letters, 31, 533-535.

BASsich, C. J., \& LudLOw, C. L. (1986). The use of perceptual methods by new clinicians for assessing voice quality. Journal of Speech \& Hearing Disorders, 51, 125-133.

Boersma, P. (1993). Accurate short-term analysis of the fundamental frequency and the harmonics-to-noise ratio of a sampled sound. Institute of Phonetic Sciences, University of Amsterdam, Proceedings, 17, 97-110.

Boersma, P., \& Weenink, D. (2008). Praat: Doing phonetics by computer (Version 4.6.09) [Computer program]. Retrieved January 4, 2009, from www.praat.org/.

Cannizzaro, M. S., Harel, B., Reilly, N., Chappell, P., \& Snyder, P. J. (2004). Voice acoustical measurement of the severity of major depression. Brain \& Cognition, 56, 30-35.

Cannizzaro, M. S., Reilly, N., Mundt, J. C., \& Snyder, P. J. (2005). Remote capture of human voice acoustical data by telephone: A methods study. Clinical Linguistics \& Phonetics, 19, 649-658. 
Carding, P. N., Carlson, E., Epstein, R., Mathieson, L., \& SHewell, C. (2001). Re: Evaluation of voice quality. International Journal of Language \& Communication Disorders, 36, 127-134.

Deliyski, D. D., Evans, M. K., \& Shaw, H. S. (2005). Influence of data acquisition environment on accuracy of acoustic voice quality measurements. Journal of Voice, 19, 176-186.

Deliyski, D. D., Shaw, H. S., \& Evans, M. K. (2005a). Adverse effects of environmental noise on acoustic voice quality measurements. Journal of Voice, 19, 15-28.

Deliyski, D. D., Shaw, H. S., \& Evans, M. K. (2005b). Influence of sampling rate on accuracy and reliability of acoustic voice analysis. Logopedics, Phoniatrics, Vocology, 30, 55-62.

Eskenazi, L., Childers, D. G., \& Hicks, D. M. (1990). Acoustic correlates of vocal quality. Journal of Speech \& Hearing Research, 33, 298-306.

Fette, B., Gibson, R., \& Greenwood, E. (1980). Windowing functions for the average magnitude difference function pitch extractor. Paper presented at the IEEE International Conference on Acoustics, Speech, \& Signal Processing.

Gerhard, D. (2003). Pitch extraction and fundamental frequency: History and current techniques (TR-CS 2003-06). Regina, SK.

Green, J. R., Beukelman, D. R., \& Ball, L. J. (2004). Algorithmic estimation of pauses in extended speech samples of dysarthric and typical speech. Journal of Medical Speech-Language Pathology, 12, 149-154.

Hirose, K., Fujisaki, H., \& Seto, S. (1992). A scheme for pitch extraction of speech using autocorrelation function with frame length proportional to the time lag. Acoustics, Speech, \& Signal Processing, 1, 149-152.

Hirst, D. (2002). Automatic analysis of prosody for multi-lingual speech corpora. In E. Keller, G. Bailly, A. Monaghan, J. Terken, \& M. Huckvale (Eds.), Improvements in speech synthesis (pp. 320-327). New York: Wiley.

John, A., Sell, D., Sweeney, T., Harding-Bell, A., \& Williams, A. (2006). The Cleft Audit Protocol for Speech-Augmented: A validated and reliable measure for auditing cleft speech. Cleft PalateCraniofacial Journal, 43, 272-288.

Karnell, M. P., Hall, K. D., \& Landahl, K. L. (1995). Comparison of fundamental frequency and perturbation measurements among three analysis systems. Journal of Voice, 9, 383-393.

Karnell, M. P., Scherer, R. S., \& Fischer, L. B. (1991). Comparison of acoustic voice perturbation measures among three independent voice laboratories. Journal of Speech \& Hearing Research, 34, 781-790.

KENT, R. D. (1996). Hearing and believing: Some limits to the auditoryperceptual assessment of speech and voice disorders. American Journal of Speech-Language Pathology, 5, 7-23.
KEnt, R. D., \& READ, C. (2002). Acoustic analysis of speech. San Diego: Singular Thomson Learning.

Kent, R. D., Vorperian, H. K., \& Duffy, J. R. (1999). Reliability of the multi-dimensional voice program for the analysis of voice samples of subjects with dysarthria. American Journal of Speech-Language Pathology, 8, 129-136.

Mendoza, E., Muñoz, J., \& Valencia Naranjo, N. (1996). The longterm average spectrum as a measure of voice stability. Folia Phoniatrica et Logopaedica, 48, 57-64.

Morris, R. J., \& Brown, W. S. (1996). Comparison of various automatic means for measuring mean fundamental frequency. Journal of Voice, 10, 159-165.

Mueller, P. B. (1997). The aging voice. Seminars in Speech \& Language, 18, 159-168.

Mundt, J. C., Snyder, P. J., Cannizzaro, M. S., Chappie, K., \& GerALTS, D. S. (2007). Voice acoustic measures of depression severity and treatment response collected via interactive voice response (IVR) technology. Journal of Neurolinguistics, 20, 50-64.

Perry, C. K., Ingrisano, D. R., \& Scott, S. R. (1996). Accuracy of jitter estimates using different filter settings on Visi-Pitch: A preliminary report. Journal of Voice, 10, 337-341.

RABINER, L. R. (1977). On the use of autocorrelation analysis for pitch detection. IEEE Transactions on Acoustics, Speech, \& Signal Processing, 25, 24-33.

Scherer, R. C., VAIL, V. J., \& GuO, C. G. (1995). Required number of tokens to determine representative voice perturbation values. Journal of Speech \& Hearing Research, 38, 1260-1269.

Takagi, T., Seiyama, N., \& Miyasaka, E. (2000). A method for pitch extraction of speech signals using autocorrelation functions through multiple window lengths. Electronics \& Communications in Japan, 83, 67-79.

Titze, I. R. (1994). The G. Paul Moore lecture: Toward standards in acoustic analysis of voice. Journal of Voice, $8,1-7$.

Titze, I. R., \& Liang, H. (1993). Comparison of $F_{\mathrm{o}}$ extraction methods for high-precision voice perturbation measurements. Journal of Speech \& Hearing Research, 36, 1120-1133.

Vogel, A. P., \& Maruff, P. (2008). Comparison of voice acquisition methodologies in speech research. Behavior Research Methods, 40, 982-987.

Zraick, R. I., Wendel, K., \& Smith-Olinde, L. (2005). The effect of speaking task on perceptual judgment of the severity of dysphonic voice. Journal of Voice, 19, 574-581.

(Manuscript received June 18, 2008;

revision accepted for publication October 29, 2008.) 